\title{
Research report \\ Testicular hormones do not regulate sexually dimorphic Pavlovian fear conditioning or perforant-path long-term potentiation in adult male rats
}

\author{
Stephan G. Anagnostaras ${ }^{a, c, *}$, Stephen Maren d, Joseph P. DeCola a Nathan I. Lane ${ }^{\text {b,c }}$, \\ Greg D. Gale ${ }^{a}$, Barney A. Schlinger ${ }^{\mathrm{b}, \mathrm{c}}$, Michael S. Fanselow ${ }^{\mathrm{a}, \mathrm{c}}$ \\ ${ }^{a}$ Department of Psychology, The University of California, 405 Hilgard Ave., Los Angeles, CA 90095-1563, USA \\ ${ }^{\mathrm{b}}$ Department of Physiological Science, The University of California, Los Angeles, CA 90095, USA \\ ${ }^{c}$ Brain Research Institute, The University of California, Los Angeles, CA 90095, USA \\ ' Department of Psychology and Neuroscience Program, The University of Michigan, Ann Arbor, MI 48109-1109, USA
}

Received 5 April 1997; received in revised form 17 May 1997; accepted 17 July 1997

\begin{abstract}
We recently reported that Pavlovian fear conditioning and hippocampal perforant-path long-term potentiation (LTP) are sexually dimorphic in rats. Males show greater contextual fear conditioning, which depends on the hippocampus, as well as greater hippocampal LTP. In order to examine the role of circulating gonadal hormones in adult male rats, animals were castrated in two experiments, and Pavlovian fear conditioning and in vivo perforant-path LTP were examined. It was found that sexually-dimorphic LTP and fear conditioning are not regulated by the activational effects of testicular hormones in adult male rats. That is, in every respect, castrated male rats were similar to intact male rats in Pavlovian fear conditioning and hippocampal LTP. It is likely that sexual dimorphism in this system is established earlier in development by the organizational effects of gonadal hormones. 1998 Elsevier Science B.V. All rights reserved.
\end{abstract}

Keywords: Hippocampus; LTP; Testosterone; Sex differences; Learning; Spatial; Context

\section{Introduction}

Several forms of hippocampal-dependent learning and memory are sexually dimorphic in rats. For example, we found a selective sexual dimorphism in contextual, but not auditory cue, Pavlovian fear conditioning [14]. This is consistent with several reports of sex differences in spatial learning such as the radial arm and Morris water mazes where differences are typically attributed to sexually-dimorphic hippocampal anatomy $[4,7,8,21-23]$, cf. [26-28]. These sex differences may

\footnotetext{
* Corresponding author. Tel.: + 1310 82:27100; fax: +1 310 2065895; e-mail: stephan@lifesci.ucla.edu
}

have parallels with sexually-dimorphic cognitive performance in verbal and spatial reasoning in humans [26].

We have also recently discovered a robust sex difference in hippocampal long-term potentiation (LTP), a prominent cellular model of associative learning and memory in animals [13]. Male rats exhibit greater in vivo perforant path-granule cell LTP than female rats $[14,16]$. Because this parallels the difference in contextual fear conditioning, which seems to require LTP induction $[6,10]$, we have argued that sex differences in hippocampal-dependent learning may be a consequence of sexually-dimorphic LTP $[14,16]$. It is then particularly germane to investigate the origin of these sex differences. If sexually-dimorphic performance on these 
tasks is the result of the sex difference in hippocampal LTP, then manipulating the factors that regulate sexually-dimorphic synaptic plasticity should cause parallel changes in the behavioral sex differences.

Many years of research on sexual behavior in rodents have established fundamental views for the hormonal regulation of sex differences in behavior [20]. Structural sex differences in the central nervous system often result from organizational effects of the presence (or absence) of testosterone (and its metabolites) during certain critical periods in development $[1,2]$. Once this sexually-dimorphic foundation is established, sex-specific behaviors may then be activated by the presence of testicular or ovarian factors. Thus sex differences in LTP and contextual fear may be due to organizational effects of gonadal steroids and/or activational effects of testicular or ovarian factors in adult rats.

As a first step in finding the basis of sexually-dimorphic LTP and fear conditioning, we chose to examine the possible activational role of testicular factors. The acquisition and extinction of Pavlovian fear conditioning and in vivo perforant path-granule cell LTP were examined in castrated and intact (or testosterone replaced) rats.

\section{Materials and methods}

\subsection{Experiment 1. The effect of castration on rate of conditioning and extinction in male rais}

\subsubsection{Subjects}

For Exp. 1, 20 male Long-Evans (Blue-Spruce derived) rats (110 days) bred at UCLA were used. They were individually housed in metal cages located in a colony maintained on a 14:10-h light/dark cycle. They had unrestricted access to food and water and were handled prior to and after surgery.

\subsubsection{Surgery}

The animals were divided into three groups: (1) no surgery was performed on Unoperated animals $(n=4)$, (2) Cast + Blank animals $(n=8)$ were given ether anesthesia, castrated according to standard surgical protocol, and given a blank silastic implant, and (3) Cast + Test animals $(n=8)$ were given ether anesthesia, castrated and then given a testosterone-filled silastic implant ( $1.59 \mathrm{~mm}$ inner diameter, $3.2 \mathrm{~mm}$ outer diameter, $45 \mathrm{~mm}$ long). Silastic implarits were soaked overnight in $0.01 \mathrm{M}$ phosphate buffer and implanted s.c. in the neck. Testosterone implants of this type effectively achieve physiological testosterone concentration in the blood of castrated male rats [11].

\subsubsection{Training}

Twenty days after surgery, animals were given one training shock per day for three consecutive days. On each day, the animals were transported to the laboratory and immediately placed into conditioning chambers. After $4 \mathrm{~min}$, the animals received one, $1 \mathrm{~s}, 0.5 \mathrm{~mA}$ unsignaled scrambled footshock, and returned to their home cages $30 \mathrm{~s}$ later. Freezing behavior, an index of conditional fear in the rat [5], was scored blind using an 8 -s time sampling procedure. For each rat, 32 observations during the 4-min interval prior to the shock on each day were converted into percent of time freezing measures to form an acquisition function. After training, the animals received three days of extinction, each of which consisted of the animals being placed in the original training context for $8 \mathrm{~min}$. Sixty-four observations per rat per day were converted into freezing measures.

\subsubsection{Contexts.}

The training and test environment consisted of aluminum (side walls) and Plexiglas (front, back, and top) chambers $(28 \mathrm{~W} \times 21 \mathrm{H} \times 22 \mathrm{~cm} \mathrm{D}$; Lafayette Instruments, Lafayette, IN). The floor of each chamber had 18 steel rods $(0.2 \mathrm{~cm}$ radius, $1.5 \mathrm{~cm}$ apart $)$ connected to a shock scrambler and generator. The shock generators along with fans inside each chamber supplied background noise of $70 \mathrm{~dB}$. The chambers were scented with a $5 \%$ ammonia solution (in collection pans below the rods). These chambers were in a well-lit room separate from the observers, who viewed the animals on video monitors.

\subsection{Experiment 2. The effect of castration on sexually-dimorphic fear conditioning and perforant-path LTP}

\subsubsection{Subjects}

For Exp. 2, 61 Long-Evans (Blue-Spruce derived) rats ( $90-120$ days) bred at UCLA were used. They were individually housed in metal cages located in a colony maintained on a 14:10-h light/dark cycle. They had unrestricted access to food and water and were handled prior to and after surgery.

\subsubsection{Castration}

The animals were subdivided into groups as follows: (1) Unoperated Males $(n=16)$ were not subjected to any surgical procedure during this phase, (2) Sham Males $(n=9)$ were anesthetized with Nembutal (sodium pentobarbital), $65 \mathrm{mg} / \mathrm{kg}$, i.p., with $0.04 \mathrm{mg} / \mathrm{kg}$ atropine $\mathrm{HCl}$, i.p, and wound clips were placed onto the skin outside of their testicles, (3) Cast Males $(n=10)$ were given anesthesia and castrated, (4) Unoperated Females $(n=16)$ were not subjected to surgery, and (5) Sham Females $(n=10)$ were given anesthesia $(45 \mathrm{mg} / \mathrm{kg} \mathrm{Nem}$ - 
butal with atropine) and wound clips were placed on the skin between the urethra and the anus. It was our intention that Unoperated and Sham animals would be collapsed, given the absence of behavioral differences, into 'intact' groups.

\subsubsection{Behavioral training}

Three weeks after surgery, all of the animals were given one signaled fear conditioning session which consisted of three tone-shock pairings. The animals were transported to the laboratory and immediately placed into conditioning chambers. After $2 \mathrm{~min}$, a $30-\mathrm{s}, 2 \mathrm{kHz}$, $90 \mathrm{~dB}$ (A-scale) tone was presented that coterminated with a 2-s, $1 \mathrm{~mA}$ scrambled footshock. Sixty-four s later animals received another tone-shock pairing and this was repeated until the animals had received three pairings. One min after the last trial, the animals were removed and returned to their home cages. Freezing behavior was scored (as in Exp. 1) during the initial 2 min of conditioning (prior to the first shock) in order to assess the baseline fear response to this context.

\subsubsection{Behavioral testing}

On the next day, all of the animals were given a context fear test; the animals were placed in the original training chambers for $4 \mathrm{~min}$. One day later, the animals were brought to a novel context (described below) for a 4-min 'off-baseline' tone test. The rats were placed in the novel chambers, and after a 2-min baseline interval, the tone (as before) was presented for $2 \mathrm{~min}$. Freezing was scored continuously by blind observers using an 8-s time sampling procedure during both tests.

\subsubsection{Contexts}

The training and context test environments were those used in Exp. 1. During training, tones were presented from a speaker in the wall of each conditioning chamber. The tone test context was in a separate room. The chambers (same dimensions as above) in this room had a white rear wall and two white plastic side walls $(24 \times 21 \mathrm{~cm})$ placed at $60^{\circ}$ to the floor forming a triangular enclosure. The floors consisted of 17 staggered rods (two rows $1 \mathrm{~cm}$ vertically apart; for each row, $2.6 \mathrm{~cm}$ apart). Background white noise $(70 \mathrm{~dB})$ was supplied by a noise generator and the chambers were scented with a $1 \%$ acetic acid solution. This room was kept entirely dark except for a $60 \mathrm{~W}$ red light bulb. The carriers used to transport the animals to each context were also different. Animals show little generalization between these contexts (Fig. 3C, tone baseline).

\subsubsection{Acute electrophysiology}

In order to determine if testicular hormones regulate hippocampal LTP in the adult male rat, perforant path-dentate granule cell synaptic plasticity was examined in a representative subset of animals from the Cast
Male $(n=6)$ and Sham Male $(n=7)$ groups in Exp. 2. Electrophysiological testing took place $2-3$ weeks after the last behavioral testing session. The rats were anesthetized with urethane (ethyl carbamate, $1.6 \mathrm{~g} / \mathrm{kg}$, i.p.) and implanted using stereotaxic techniques unilaterally with a stimulating electrode in the perforant path and a recording electrode in the ipsilateral dentate gyrus. The recording electrode (an Epoxylite-coated stainless-steel size 00 insect pin with the $50 \mu \mathrm{m}$ tip uninsulated) was implanted in the hilus of the dentate gyrus $(3.3 \mathrm{~mm}$ posterior to bregma, $2.4 \mathrm{~mm}$ lateral to the midline, and $2.8-3.0 \mathrm{~mm}$ ventral to dura). The bipolar stimulating electrode (two Epoxylite-coated insect pins with the 500 $\mu \mathrm{m}$ tips uninsulated and separated by $1 \mathrm{~mm}$ ) was implanted in the medial perforant pathway $(8.1 \mathrm{~mm}$ posterior to bregma, $4.4 \mathrm{~mm}$ lateral to the midline, and $2.0-4.0 \mathrm{~mm}$ ventral to dura). The ventral locations of both electrodes were adjusted to maximize perforant path-evoked dentate hilar field potentials. In prior studies using these techniques, histological verification has revealed a $100 \%$ placement success rate $[14,16]$. Reference and ground electrodes were attached to stainless steel skull screws affixed to the skull over the nasal sinus. Body temperature was maintained at a constant $37^{\circ} \mathrm{C}$ with a heating pad.

Extracellular dentate hilar field potentials evoked by single-pulse perforant path stimulation $(100-\mu$ s pulses) were amplified (gain $=100)$, bandpass filtered $(1 \mathrm{~Hz}-5$ $\mathrm{kHz}$ ), displayed on an oscilloscope, and digitized and written to disk by a computer (DataWave Systems, Longmont, $\mathrm{CO}$ ).

After stable hilar field potentials had been observed for at least $30 \mathrm{~min}$, perforant path-evoked field potentials (stimulation current, 200-600 $\mu \mathrm{A}$, adjusted to elicit a $3-\mathrm{mV}$ population spike) were sampled and recorded every $20 \mathrm{~s}$ during a 10 -min period before and a 60-min period after perforant path high-frequency stimulation (HFS). HFS consisted of ten pairs of 400$\mathrm{Hz}$ bursts (burst duration $=25 \mathrm{~ms}$, ten pulses per burst) delivered at the same current used for baseline recording. Bursts within a pair were delivered at theta rhythm ( $5 \mathrm{~Hz}$, interburst interval $=200 \mathrm{~ms}$ ) and each of the ten pairs of bursts was separated by $10 \mathrm{~s}$.

\subsubsection{Radioimmunoassays ( $R I A S$ )}

After electrophysiological testing was completed, heart blood was drawn from the animals via a $1-\mathrm{ml}$ syringe containing heparin $(0.1 \mathrm{ml})$. The blood was transferred to a $10-\mathrm{ml}$ tube and centrifuged $(2000 \mathrm{rpm})$ for $20 \mathrm{~min}$ to isolate the plasma supernatant. After centrifugation, the plasma was drawn from the tubes and frozen. Shortly thereafter, plasma testosterone (T) levels were analyzed using the Diagnostic Products Corporation (Los Angeles, CA) total testosterone coata-count kit. The kit is a solid-phase RIA, in which the T-specific antibody is immobilized to the walls of 

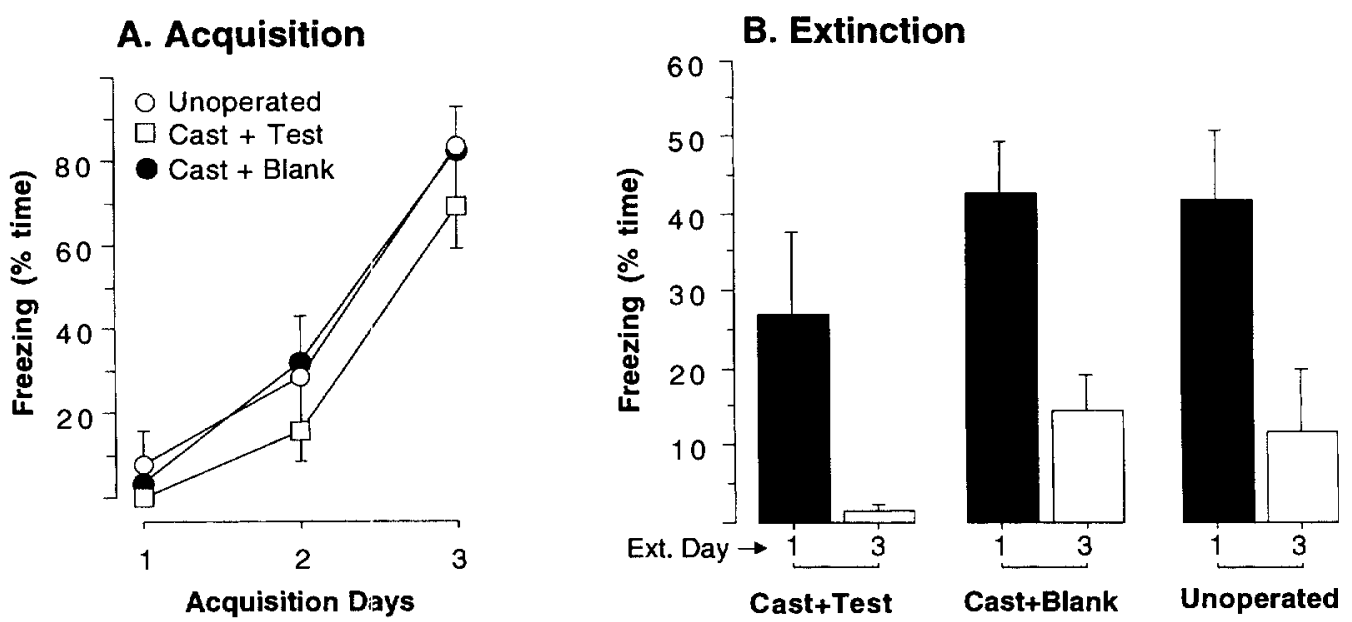

Fig. 1. Experiment 1: Acquisition and extinction of contextual fear. (A) Acquisition. Contextual fear (mean $\%$ time spent freezing \pm SEM) was assessed during the 4-min interval prior to each shock on three consecutive acquisition days. (B) Extinction. The mean freezing during the entire 8-min period is shown for the first and third extinction days. It is clear from Exp. 1 that male rats which were depleted of testosterone (Cast + Blank) were comparable in terms of acquisition and extinction of contextual fear to both Unoperated and replacement therapy (Cast + Test) rats.

polypropylene test tubes. The antiserum is highly specific for $\mathrm{T}$, with minimal crossreactivity to other compounds possibly present in the plasma. For example, crossreactivity with dihydrotestosterone, its closest analog, is less than $5 \%$. Unextracted rat plasma was added to the polypropylene tubes followed by the addition of ${ }^{125}$ I-labeled T. Following a $3-\mathrm{h}$ incubation, during which labeled $\mathrm{T}$ competes with unlabeled $\mathrm{T}$ for antibody sites, the polypropylene tubes were decanted to separate bound from free hormone. The tubes were counted in a gamma counter and steroid levels were determined from a calibration curve. To monitor assay precision, we included several human serum-based calibrators (provided in the kit) with the following $\mathrm{T}$ concentrations: $0,0.2,1.0 .4 .0,8.0$ and $16.0 \mathrm{ng} / \mathrm{ml}$. We prepared and included eight additional tubes containing 0 or $1 \mathrm{ng} \mathrm{T} / \mathrm{ml}$ human-serum. The average values measured for these tubes were 0.100 and 1.1132 , respectively. Further validation studies involved the addition of $500 \mathrm{pg} \mathrm{T}(2.50 \mathrm{ng} / \mathrm{ml} /$ tube $)$ to unextracted and untreated avian serum samples. Basal levels were determined to be $3.4845 \mathrm{ng} / \mathrm{ml}$; tubes containing added $\mathrm{T}$ measured $6.0834 \mathrm{ng} / \mathrm{ml}$. The upper and lower limits of detection were determined to be $10.00 \mathrm{ng} / \mathrm{ml}$ and 0.171 $\mathrm{ng} / \mathrm{ml}$, respectively. The within assay C.V. was $4.6 \%$. T values below the measurable limit $(0.171 \mathrm{ng} / \mathrm{ml})$ were conservatively assigned values of 0.171 for the purpose of statistical analysis. The $\mathrm{T}$ levels measured in these tubes accurately reflected the experimental manipulations performed confirming the procedure's accuracy over a wide range of $\mathrm{T}$ values.

\section{Results}

\subsection{Exp. 1. Acquisition and extinction of contextual fear conditioning in gonadectomized male rats}

\subsubsection{Acquisition}

Fig. 1A shows that all three groups rapidly acquired contextual fear during training and did not differ from one another. A two-factor (GROUP, DAY) multivariate analysis of variance (MANOVA) confirmed the general appearance of the graphs [GROUP $\times$ DAY interaction, $F(4,34)<0.5, \quad P>0.5$; main effect of GROUP, $F(2,17)=1.4, P>0.2]$. Paired two-tailed $t$ tests comparing the scores on day 1 and day 3 revealed that all groups showed strong evidence of significant learning [Cast + Blank, $t(7)>10, P<0.0001$; Cast + Test, $t(7)>6.7, P<0.001$; Unoperated, $t(3)>10, P<$ $0.01]$. Thus castration does not affect the acquisition of contextual fear conditioning, which we have previously shown to be sexually dimorphic $[14,16]$ (Fig. 3A).

\subsubsection{Extinction}

Fig. 1B shows that all animals rapidly extinguished contextual fear and did not differ in this respect [MANOVA: GROUP $\times$ DAY, $F(2,17)<0.7, P>0.5$; GROUP, $F(2,17)=1.5, P>0.2]$. All groups showed evidence of significant extinction [extinction day 1 versus 3, paired two-tailed $t$-tests: Cast + Blank, $t(7)>2.8$, $P<0.03$; Cast Test, $t(7)>2.3, P<0.05$; Unoperated, $t(3)>7.9, P<0.01]$. 
3.2. Exp. 2. The effect of castration on sexually-dimorphic fear conditioning and perforant-path $L T P$

\subsubsection{Radioimmunoassays}

RIA revealed that most castrations were successful in eliminating plasma testosterone. For Sham Male rats $(n=7)$, the mean level of $\mathrm{T}$ was $1.9 \mathrm{ng} / \mathrm{ml}$ (range = 1.2-3.7). Four out of the six Cast Male rats had levels of $\mathrm{T}$ below the measurable limit $(0.171 \mathrm{ng} / \mathrm{ml})$, one additional castrated rat had a $\mathrm{T}$ level below the range of that for Sham Males $(0.98 \mathrm{ng} / \mathrm{ml})$ and one had fairly normal levels of $\mathrm{T}(2.25 \mathrm{ng} / \mathrm{ml})$. For this reason, the latter rat was excluded from all further analyses, although inclusion of this rat would not affect any of the statistical conclusions. After exclusion of this rat, the mean level of $\mathrm{T}$ for Cast Male rats $(n=5)$ was 0.33 $\mathrm{ng} / \mathrm{ml}$ (depicted in Fig. 2). A Mann--Whitney $U$-test $(U=0, P<0.01)$ confirmed that castration severely depleted plasma testosterone.

\subsubsection{Behavior}

In order to simplify data analysis, we first examined whether or not Unoperated and Sham animals differed from each other on any of the behavioral measures for both Male and Female animals. Unoperated and Sham animals were compared using separate (male and female) pairwise multivariate analyses of variance (MANOVAs) with all four behavioral measures (Context Baseline, Context Test, Tone Baseline, and Tone Test). In all cases, Sham animals did not differ from Unoperated animals on these measures [Males, main effect of GROUP (sham, unoperated), $F(1,23)<0.5$, $P>0.5$; GROUP $\times$ TEST interaction, $F(3,69)<0.5$,

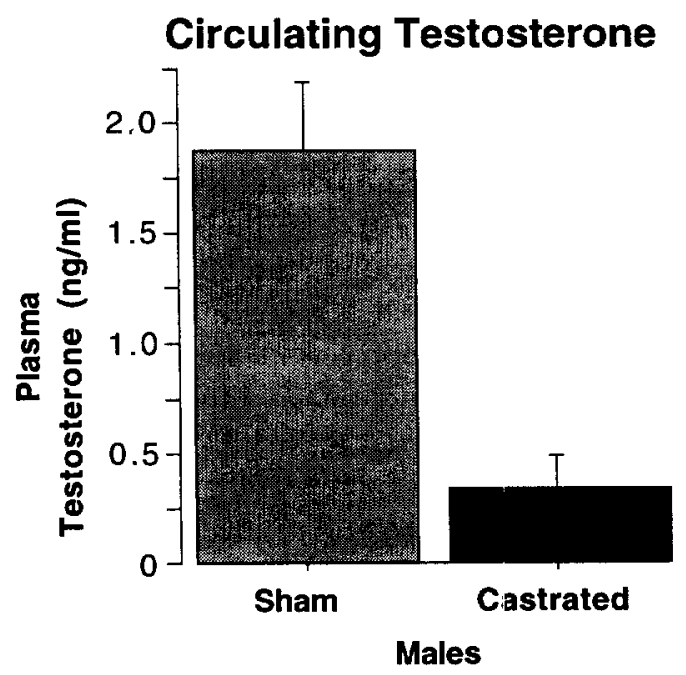

Fig. 2. Experiment 2: Plasma testosterone levels. Mean ( \pm SEM) plasma testosterone levels revealed by radioimmunoassay (RIA) after Castration or Sham surgery. RIAs clearly showed that castrations depleted plasma testosterone indicating successful surgery.
$P>0.5)$; Females, GROUP, $F(1,24)<0.5, \quad P>0.5$; GROUP $\times$ TEST interaction, $F(3,72)<0.5, P>0.5)$ ]; for this reason, Sham and Unoperated animals were collapsed for each sex in all subsequent behavioral analyses forming an Intact Female group $(n=26)$ and an Intact Male group $(n=25)$.

3.2.2.1. Contextual fear conditioning. Fig. 3A shows that animals exhibited almost no baseline freezing upon initial placement into this context prior to any conditioning during the training day (group means: Female, $0 \pm 0 \%$; Intact Male, $0.50 \pm 0.35 \%$; Cast Male, $0 \pm 0 \%$ ). In order to unambiguously examine fear induced by conditioning, baseline freezing to this context was subtracted from the raw freezing scores obtained during the context test (Fig. 3A) in order to reveal contextual fear for each animal (Fig. 3B). Females exhibited considerably less contextual fear than Intact Male or Cast Male rats when placed back in the original training chambers after a fear conditioning session. An overall univariate ANOVA confirmed group differences $[F(2,57)=6.1, P<0.01]$. Female rats froze significantly less than Cast Male (Fisher's PLSD, $P<0.02$ ) or Intact Male $(P<0.01)$ rats which did not differ from each other $(P>0.5)$. Thus, although there is a robust sex difference in contextual fear conditioning, it is not mediated by circulating testicular factors in the adult male rat.

3.2.2.2. Tone conditioning. Fig. $3 \mathrm{C}$ depicts freezing that was observed during the entire 4-min Tone Test. During the 2-min baseline period prior to the presentation of the tone, the animals showed some freezing to the novel context, and also differed significantly in this respect [ANOVA, $F(2,57)=5.3, P<0.01]$. We typically do not observe this, although the fact that it was significant is probably attributable to our unusually large sample sizes. Baseline freezing to a novel context is not readily interpretable, but it probably represents either unconditional fear to the novel context or generalized fear from the training context. Because Female rats tended to freeze less during both the baseline and tone periods, we tend to think this probably represents generalization of some contextual fear of the original training context due to slight similarities between the two environments. It is unlikely, however, that this freezing represents a response tendency for Intact Males to freeze to any condition, since they exhibit no baseline freezing to the training context (Fig. 3A). Nonetheless, in order to unambiguously examine fear of the training tone, we subtracted each animal's baseline freezing from freezing that occurred during the tone (Fig. 3C). This adjusted tone fear is depicted in Fig. 3D. The animals did not differ in terms of this tone fear $[F(2,57)=2.1, P>0.1]$. This replicates our prior report that although female rats show similar levels of tone conditioning as male rats, they exhibit less contextual 
A. Context Test

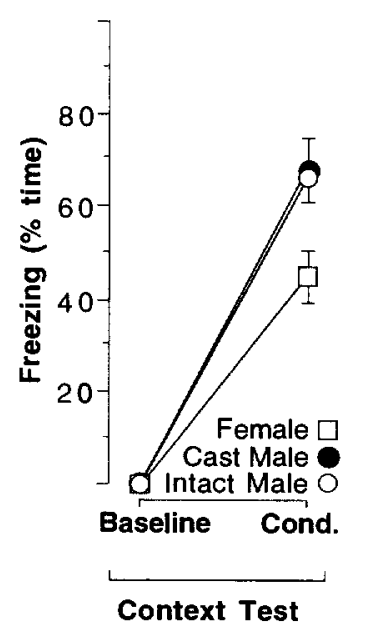

B. Contextual Fear

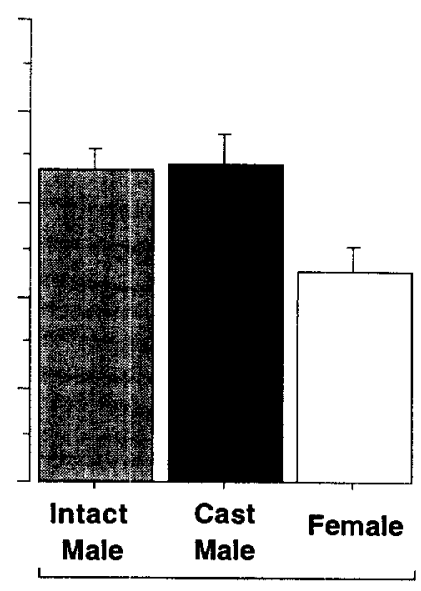

(Context - Baseline)

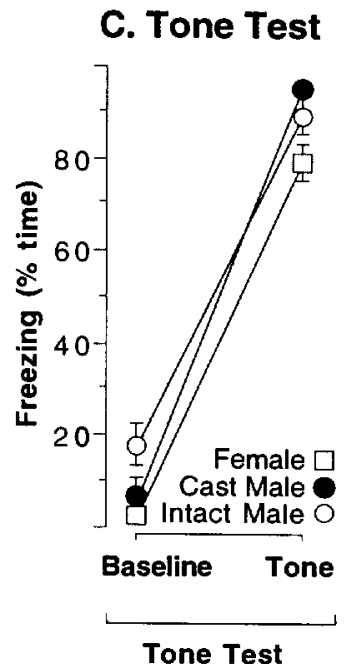

Tone Test
D. Tone Fear

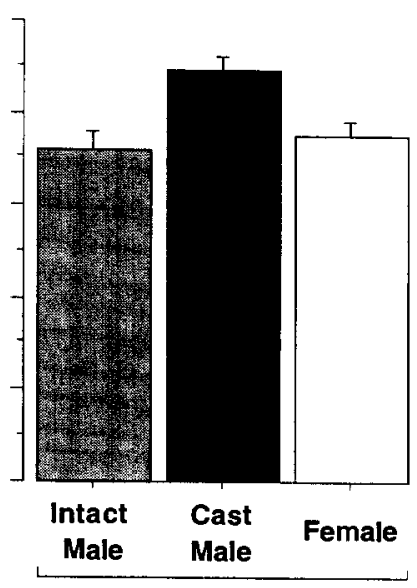

(Tone - Baseline)

Fig. 3. Experiment 2: The effect of castration on sexually-dimorphic fear conditioning. (A) Context baseline. After initial placement into the training context, but prior to any shocks, a 2-min contextual fear baseline was taken by scoring freezing behavior ( $\%$ time, mean \pm SEM). Context test: One day after a fear conditioning session that consisted of three tone-shock pairings, the animals were placed in the original training context and freezing behavior ( $\%$ time, mean \pm SEM) was scored for $4 \mathrm{~min}$. (B) Contextual fear. In order to unambiguously examine contextual fear, the context baseline was subtracted from the scores obtained during the context test. Female rats, exhibited significantly less fear of the context than did Castrated Male or Intact Male rats, which did not differ from each other. Thus, adult circulating testosterone does not mediate sexually-dimorphic contextual fear conditioning. (C) Tone test. One day after the context test, the animals were placed in a novel context, and after a 2-min baseline period, the original trainirig tone was played continuously for $2 \mathrm{~min}$. Because the groups differed slightly in terms of freezing during the baseline, raw data from the tone period cannot be interpreted. (D) Tone fear. In order to unambiguously interpret tone fear, freezing during the baseline period was subtracted from freezing during the period that the tone was on to yield adjusted tone fear. The groups did not differ significantly in this respect, although there was a modest trend for Castrated Male animals to show somewhat greater fear of the auditory cue.

fear conditioning. Moreover, castration does not seem to significantly affect fear conditioning to a tone stimulus, although examination of Fig. 3D reveals a trend toward an elevated level of tone fear.

\subsubsection{Electrophysiology}

Perforant-path LTP was assessed in Sham and Cast Male rats by recording extracellular field potentials in the dentate gyrus during a 10 -min period prior to, and a 60-min period after HFS delivered to the ipsilateral perforant path. Fig. 4A shows the average waveforms recorded from individual representative rats during the 10-min period prior to (dashed lines) and the 50-60min interval after induction of LTP (solid lines). As can be seen in Fig. 4A, these field potentials were characterized by a gradual positive-going field excitatory postsynaptic potential (EPSP) with a sharp negative-going population spike (PS) superimposed on the rising phase of the EPSP. The population EPSP reflects synaptic currents at perforant path-dentate granule cell synapses in stratum moleculare, whereas the PS reflects the synchronous action potential discharge of granule cell bodies in the stratum granulosum. The EPSP slope and PS amplitude of waveforms sampled every $20 \mathrm{~s}$ were measured and the values were normalized to the average of the 10-min baseline. Fig. 4B and C show the percentage of change relative to this baseline for the EPSP slope and PS amplitude averaged across 5-min intervals. It is obvious from visual examination of Fig. $4 \mathrm{~B}$ and $\mathrm{C}$ that perforant-path HFS induced a robust and long-lasting LTP of both EPSP slope and PS amplitude in both Sham and Castrated rats. In order to compare the two groups, these values were subjected to two separate (for EPSP slope and PS amplitude) MANOVAs which confirmed the absence of group differences [EPSP slope: GROUP $\times$ TEST interaction, $F(13,130)<0.1$; $P>0.5$; main effect of GROUP, $F(1,10)<0.1, P>$ 0.5 ; PS amplitude: GROUP $\times$ TEST, $F(13,130)<1$, $P>0.5$; GROUP, $F(1,10)<1, P>0.4$ ]. To verify the presence of long-lasting LTP, the 10-min baseline period was averaged (for each rat) and compared to the average of the 50-60-min period after HFS (for each rat) [paired two-tailed $t$-tests: EPSP slope: Sham, $t(6)=5.1, P<0.01$; Cast, $t(4)=8.3, P<0.01$; PS amplitude: Sham, $t(6)=6.1, P<0.001$; Cast, $t(4)=6.6$, $P<0.01]$. Using identical procedures to these, we have previously shown that females show much less EPSP slope LTP (but not PS amplitude LTP) than males $[14,16]$. These data clearly show that sexually-dimorphic hippocampal LTP, as with the sex difference in hippocampal-dependent contextual fear conditioning, is not mediated by circulating testosterone in the adult male rat. 
A.

Sham

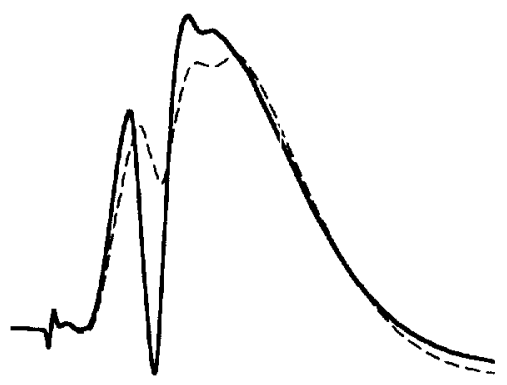

B. EPSP Slope LTP

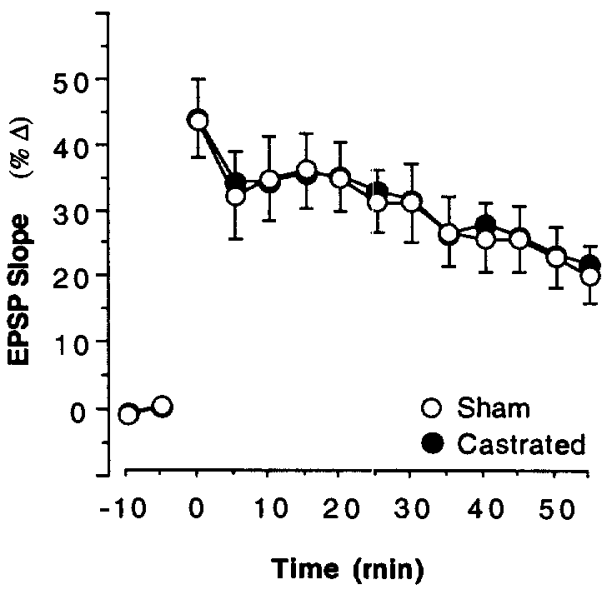

Castrated

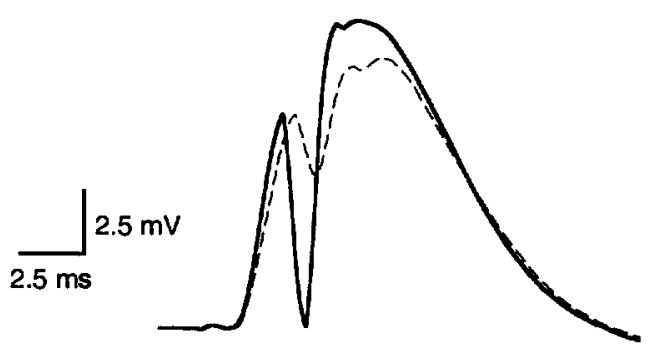

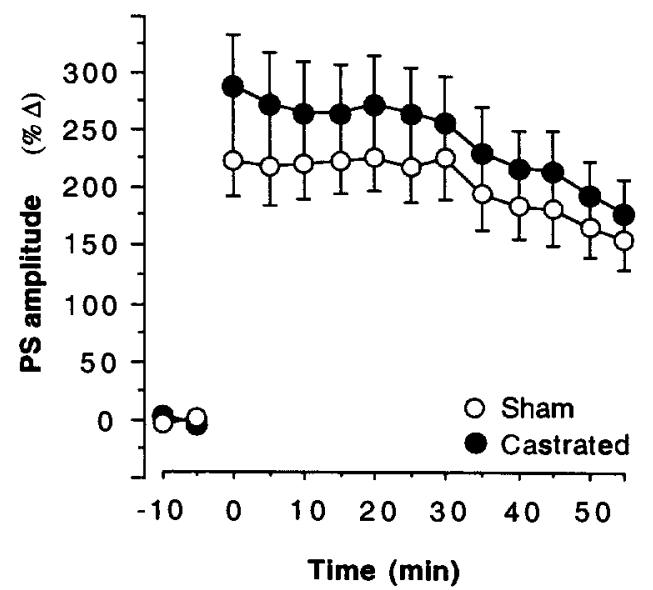

Fig. 4. Experiment 2: The effect of castration on perforant path-dentate granule cell LTP. (A) Representative waveforms. Averaged extracellular field potentials evoked in the dentate gyrus by single-pulse stimulation of the perforant path in representative Castrated and Sham male rats. Waveforms are $10 \mathrm{~min}$ averages of field potentials collected $10 \mathrm{~min}$ before and 50-60 min following perforant path LTP induction. Pre- (dotted line) and post- (solid line) LTP responses are superimposed. (B) EPSP Slope LTP. Mean ( \pm SEM) percentage of change (relative to baseline) in EPSP slope for the 70-min recording period. Perforant path HFS was delivered at $t=0$. Both groups show obvious, long-lasting EPSP LTP. Sham and Castrated rats are virtually identical, whereas, we have previously shown that female rats show less EPSP slope LTP. (C) Population Spike LTP. Mean percentage of change in Population Spike amplitude for the 70-min period. Although both groups showed significant PS amplitude and EPSP slope LTP, Sham and Castrated animals did not differ on either PS amplitude or EPSP slope LTP. Thus, circulating testosterone in the adult rat does not regulate the sex difference in perforant-path EPSP LTP.

\section{Discussion}

In the present study, castration of adult male rats had no measurable effect on sexually-dimorphic hippocampal-dependent contextual fear conditioning, or perforant-path hippocampal LTP. Although castration severely depleted serum testosterone levels, contextual fear conditioning and hippocampal LTP in castrated animals remained comparable to that of intact male rats and was well above that of female rats.

In our prior reports, male rats acquired contextual fear (but not tone fear) more rapidly than female rats, and also showed greater perforant-path granule cell hippocampal LTP, specific to the field EPSPs $[14,16]$. This is interesting, because lesions of the hippocampus produce an acquisition deficit [19], and a dense, but time-limited retrograde amnesia specific to contextual fear [9]. Moreover, administration of the NMDA receptor antagonist APV, which blocks the induction of LTP $[13,18]$, also blocks contextual fear conditioning $[6,10]$. Furthermore, there is a high correspondence between hippocampal theta rhythm and the acquisition of contextual fear [15]. Because there was a corresponding sex difference in burst depolarization evoked during HFS, and the sex difference in LTP is specific to field EPSPs, we have argued that weaker LTP was induced in females because of weaker NMDA receptor activation [14]. Thus, it is possible that females show less contextual fear conditioning, because of their weaker NMDA receptor-dependent LTP.

These experiments suggest that sexually-dimorphic fear conditioning and hippocampal LTP are not regulated by activational effects of testosterone, its metabolites, or other testicular factors in the adult male rat. It 
is more likely that these sex differences are caused by organizational effects of testosterone early in development. For example, it has been reported that normal male rats perform better than normal female rats on both the radial arm and Morris water mazes, while early testosterone administration reverses this sex difference and induces a more 'male-like' hippocampus in females $[21,22,27]$. This sex difference has been attributed to structural differences in the granule cell layer of the hippocampus [21].

Likewise, administration of estradiol or the aromatase inhibitor ATD during the first 10 postnatal days masculinizes adult learning on the radial arm maze $[26,27]$. As with reproductive behavior, male spatial behavior appears to be organized by the presence of estradiol in the brain early in development. Given the presence of aromatase activity in the hippocampus early in rat development, the possibility that estradiol organizes the hippocampus is quite likely [12]. Indeed, estradiol implants aimed at the hippocampus (early in development) appear to be sufficient to reverse the detrimental effects of neonatal castration on radial arm maze performance [26]. There is, then, considerable evidence that sex differences in the anatomy and function of the hippocampus are organized perinatally by testosterone acting via aromatization to estradiol $[12,26,28]$. Nonetheless, it is not possible to generalize findings from spatial maze learning directly to contextual fear conditioning, because these are not nearly the same tasks. Although all depend on an intact hippocampus, they are also dissimilar in many respects (e.g. fear conditioning depends on the amygdala as well) and the organizational roles of these factors remain to be examined in contextual fear conditioning.

It is also possible, however, that sex differences are not due to the organizational effects of testosterone developmentally, but rather to the effects of ovarian factors acting on the hippocampus of adult female rats. For example, Warren et al. [25] recently demonstrated a relationship between phase of the estrus cycle and Schaffer collateral-CAl LTP in vivo. Female rats tested in the evening of proestrus (when estrogen levels are highest) exhibited greater EPSP slope LTP than females in estrus, diestrus, or proestrus in the morning, and even exceeded that of male rats. Interestingly, when tested in the morning, male rats exhibited the greatest CA1 LTP. This modulation of LTP by the estrus cycle is accompanied by changes in dendritic spine density and synaptic density in CAl (they are highest in proestrus) that appear to be regulated by estradiol and progesterone $[29,30]$. This is accompanied, however, by a decrease in contextual fear [17] and water maze performance [3,7], cf. [24] in rats during proestrus, and indicates a striking lack of correspondence between hippocampal LTP and hippocampal-dependent learning [17].
A number of factors might account for the negative correlation between CA1 LTP and hippocampal-dependent learning in cycling females. For instance, in both contextual fear conditioning [17] and water maze learning [3,24], estrus effects on acquisition and performance were confounded, because the rats were trained and/or tested in the same phase of the estrus cycle. Because LTP is presumed to have a selective role in the acquisition processes $[6,10]$, the effect of estrus cycle fluctuation in LTP on learning will remain unclear until animals are trained in only one phase of the cycle and then tested across every phase. A further problem in interpreting these data is that estrus cycle effects on LTP have only been examined in Schaffer collateralCA1 pyramidal cell LTP [25], whereas the sex difference we reported, which was associated with enhanced hippocampal-dependent learning, was apparent in perforant path-granule cell LTP $[14,16]$. It may be the case that perforant-path LTP does not show estrus-associated changes, as cells in the dentate gyrus seem to show little fluctuation in dendritic spine density across the estrus cycle [31]. Furthermore, it is unclear if there is a sex difference in the average male and female CAl LTP [25]. Until these issues are examined, it will remain unclear how to relate our data on parallel sex differences in perforant-path LTP and contextual fear conditioning, and contrasting estrus cycle effects on CAl LTP and contextual fear (or spatial learning) in female rats.

Of course, these effects may be entirely different. As suggested earlier, the sex difference we observed in perforant-path LTP and learning may be due to an organizational effect of testosterone. Variations in CA1 LTP appear to be regulated by the activational effects of ovarian steroids. In males, enhanced perforant path LTP may augment hippocampus-dependent learning, whereas enhanced CAI LTP in females may impair this form of learning. Until the exact mechanisms of sexually-dimorphic perforant-path LTP and estrus-sensitive CA1 LTP are identified it will remain unclear how they are related and what their impact on learning is (either detrimental or beneficial). Thus, it is possible that multiple hormonal effects are modulating hippocampal physiology and function; on the one hand, testosterone (or its metabolites) may be organizing, early in development, a hippocampus in the male that is more suitable for the induction of LTP and the learning of contextual fear (and spatial tasks). On the other hand, estradiol may have activational effects in adulthood that modulate hippocampal physiology and function across the estrus cycle in the female.

Considerable further research is necessary to determine the possible role(s) of gonadal factors in establishing the sex difference in perforant-path hippocampal LTP and contextual fear conditioning. Based on the present study, it can be concluded that these sexual 
dimorphisms are not caused by circulating testicular factors in the adult male rat. The next steps will be to examine ovarian factors in adult female rats, as well as the organizational roles of early hormone exposure on LTP and fear conditioning. Further examination of corresponding sex differences in learning and synaptic plasticity also promises to offer considerable insight into the relationships between these two mechanisms.

\section{Acknowledgements}

This research was supported by an NIMH grant (MH39786) to MSF. SA and SM were supported by institutional NIMH NRSA (MH15795) to UCLA. We would like to thank Jane Lubischer for her help with the surgical procedures, and greatly appreciate the thoughtful comments by Beatrice DeOca, Jennifer Sage, and two anonymous reviewers on an early version of this manuscript.

\section{References}

[1] Arnold AP, Gorski RA. Gonadal steroid induction of structural sex differences in the nervous system. Annu Rev Neurosci 1984;7:413-42.

[2] Arnold AP, Schlinger BA. Sexual differentiation of brain and behavior: the zebra finch is not just a flying rat. Brain Behav Evol 1993;42:231-41.

[3] Berry B, McMahan R, Gallagher M. Spatial learning and memory at defined points of the estrous cycle: effects on performance of a hippocampal-dependent task. Behav Neurosci 1996;111: 267-74.

[4] Bucci DJ, Chiba AA, Gallagher M. Spatial learning in male and female Long-Evans rats. Behav Neurosci 1995;109:180-3.

[5] Fanselow MS, Bolles RC. Naloxone and stock-elicited freezing in the rat. J Comp Physiol Psychol 1979;93:736-44.

[6] Fanselow MS, Kim JJ, Yipp J, DeOca B. Differential effects of an NMDA antagonist, APV, on acquisition. of fear of auditory and contextual cues. Behav Neurosci 1994; 108:235-40.

[7] Frye CA. Estrus-associated decrements in water maze task are limited to acquisition. Physiol Behav 1994;20:187-91.

[8] Juraska JM, Henderson V, Muller J. Differential rearing experience, gender, and radial maze performance. Dev Psychobiol 1984;17:209--15.

[9] Kim JJ, Fanselow MS. Modality-specific retrograde amnesia of fear. Science 1992;256:675-7.

[10] Kim JJ, DeCola JP, Landeria-Fernandez J, Fanselow MS. $N$ methyl-D-aspartate receptor antagonist APV blocks acquisition but not expression of fear conditioning. Behav Neurosci 1991; 105:126-33.

[11] Lubischer JL, Arnold AP. Evidence for target regulation of the development of androgen sensitivity in rat spinal motoneurons. Dev Neurosci 1995;17:106-17.

[12] MacLusky NJ, Clark AS, Naftolin F, Goldman-Rakic PS. Estrogen formation in the mammalian brain: possible role of aromatase in sexual differentiation of the hippocampus and neocortex. Steroids 1987;50:459-74.

[13] Maren S, Baudry M. Properties and mechanisms of long-term synaptic plasticity in the mammalian brain: relationships to learning and memory. Neurobiol Learn Memory 1995;63:1-18.

[14] Maren S, DeOca B, Fanselow MS. Sex differences in hippocampal long-term potentiation (LTP) and Pavlovian fear conditioning in rats: positive correlation between LTP and contextual learning. Brain Res 1994;661:25-34.

[15] Maren S, DeCola JP, Swain RA, Fanselow MS, Thompson RF. Parallel augmentation of hippocampal long-term potentiation, theta rhythm, and contextual fear conditioning in water-deprived rats. Behav Neurosci 1994;108:44-56.

[16] Maren S. Sexually dimorphic perforant path long-term potentiation (LTP) in urethane-anesthetized rats. Neurosci Lett 1995; 196:177-80.

[17] Markus EJ, Zecevic M. Sex differences and estrous cycle changes in hippocampus-dependent fear conditioning. Psychobiology (in press).

[18] Morris RGM, Anderson E, Lynch GS, Baudry M. Selective impairment of learning and blockade of long-term potentiation by an $N$-methyl-D-aspartate receptor antagonist, AP5. Nature 1986;319:774-6.

[19] Phillips RG, LeDoux JE. Differential contribution of amygdala and hippocampus to cued and contextual fear conditioning. Behav Neurosci 1992;106:274-85.

[20] Phoenix CH, Goy RW, Gerall AA, Young WC. Organizing action of prenatally administered testosterone proprionate on the tissues mediating mating behavior in the female guinea pig. Endocrinology 1959;93:369--82.

[21] Roof RL, Havens MD. Testosterone improves maze performance and induces development of a male hippocampus in females. Brain Res 1992;572:310-3.

[22] Roof RL. Neonatal exogenous testosterone modifies sex difference in radial arm and Morris water maze performance in prepubescent and adult rats. Behav Brain Res 1993;53:1-10.

[23] van Harren F, Wouters $M$, van den Poll NE. Absence of behavioral differences between male and female rats in different radial maze procedures. Physiol Behav 1987;39:409-12.

[24] Warren SG, Juraska JM. Spatial and nonspatial learning across the rat estrous cycle. Behav Neurosci 1997;111:258-66.

[25] Warren SG, Humphreys AG, Juraska JM, Greenough WT. LTP varies across the estrous cycle: Enhanced synaptic plasticity in proestrous rats. Brain Res 1995;703:26-30.

[26] Williams CL, Meck WH. The organizational effects of gonadal steroids on sexually dimorphic spatial ability. Psychoneuroendocrinology 1991;16:155-76.

[27] Williams CL, Barnett AM, Meck WH. Organizational effects of early gonadal secretions on sexual differentiation in spatial memory. Behav Neurosci 1990;104:84-97.

[28] Wimer RE, Wimer C. Three sex dimorphisms in the granule cell layer of the hippocampus in house mice. Brain Res 1985;328: $105-9$.

[29] Woolley CS, McEwen BS. Estradiol mediates fluctuation in hippocampal synapse density during the estrous cycle in the adult rat. J Neurosci 1992;12:2549-54.

[30] Woolley CS, McEwen BS. Roles of estradiol and progesterone in regulation of hippocampal dendritic spine density during the estrous cycle in the rat. J Comp Neurol 1993;336:293-306.

[31] Woolley CS, Gould E, Frankfurt M, McEwen BS. Naturally occurring fluctuation in dendritic spine density on adult hippocampal pyramidal neurons. J Neurosci 1990;10:4035-9. 